\title{
Prevalencia y distribución de defectos óseos en un grupo de potenciales pacientes ortodóncicos no tratados utilizando Tomografías Computarizadas Cone Beam
}

\author{
Prevalence and distribution of bone defects in a group of \\ potential untreated orthodontic patients using Cone Beam \\ Computed Tomography
}

Recibido: 2017/04/15. Aceptado: 2017/06/20. Publicado: 2017/09/01

\section{Laura De Rosa ${ }^{1}$ \\ Carolina Dueñas ${ }^{2}$}

1 Universidad San Francisco de Quito, Colegio de Ciencias de la Salud, Escuela de Odontología, Clínica Odontológica, Campus Cumbayá, casilla postal 17-1200-841, Quito-Ecuador.

Correo electrónico: Iauderosa87@gmail.com

2 Universidad San Francisco de Quito, Colegio de Ciencias de la Salud, Escuela de Odontología, Clínica Odontológica, Campus Cumbayá, casilla postal 17-1200-841, Quito-Ecuador.

Correo electrónico: carolinaduenas2001@yahoo.com 


\section{Resumen}

El diagnóstico ortodóncico requiere un examen minucioso de los tejidos de soporte periodontal; alteraciones como dehiscencias, fenestraciones y otros defectos óseos deberían ser incluidos tanto dentro del diagnóstico como dentro del plan de tratamiento.

El propósito de este estudio fue investigar la prevalencia y distribución de defectos óseos en un grupo de potenciales pacientes ortodóncicos no tratados, utilizando Tomografías Computarizadas Cone Beam (TCCB), con el objetivo de evaluar su posible incorporación a los estudios diagnósticos básicos previos al tratamiento ortodóncico.

Se analizaron 33 TCCB y un total de 792 dientes en busca de dehiscencias y fenestraciones y posteriormente se midió el grosor del hueso alveolar vestibular y palatino o lingual en el tercio medio radicular de 528 dientes. Los datos obtenidos fueron utilizados para realizar un análisis estadístico descriptivo e inferencial que arrojó los siguientes resultados: un 36\% de la muestra presentó algún tipo de defectos óseos; las dehiscencias fueron más comunes que las fenestraciones y fueron más comunes en la arcada inferior, mientras que las fenestraciones lo fueron en la superior; la cortical más gruesa se encontró en la superficie lingual de molares inferiores y la más delgada en la superficie vestibular de caninos inferiores.

Las TCCB poseen el potencial de reemplazar las radiografías convencionales y permitir realizar decisiones diagnósticas acertadas en base a la arquitectura ósea de cada paciente, por lo que se recomienda su uso como auxiliar diagnóstico, especialmente en pacientes de alto riesgo.

Palabras clave: Tomografía Computarizada Cone Beam, dehiscencia, fenestración, hueso alveolar.

\section{Abstract}

Orthodontic diagnosis requires a thorough examination of the periodontal tissues; alterations such as dehiscences, fenestrations and other bone defects should be included within the diagnosis and the treatment plan.

The purpose of this study was to investigate the prevalence and distribution of bone defects in a group of potential orthodontic patients which were untreated, using Cone Beam Computed Tomography (CBCT), in order to evaluate their possible incorporation into basic diagnostic studies prior to orthodontic treatment.

The presence of dehiscences and fenestrations was analyzed in a total of 33 CBCTs and 792 teeth, and the thickness of the vestibular and palatal or lingual alveolar bone was measured in the middle third of 528 teeth. The data obtained were used to perform a descriptive and inferential statistical analysis that yielded the following results: $36 \%$ of the sample presented some type of bone defects; dehiscences were more common than fenestrations and were more common in the lower arch, while fenestrations were in the upper; the thicker cortical bone was found on the lingual surface of lower molars, and the thinner cortical bone on the buccal surface of lower canines.

As CBCTs have the potential to replace conventional radiographs and allow accurate diagnostic decisions based on each patient's bone architecture, I recommend their use as a diagnostic aid, especially in high-risk patients.

Key words: Cone Beam Computed Tomography, dehiscence, fenestration, alveolar bone. 


\section{Introducción}

La integridad del hueso alveolar es de suma importancia para el tratamiento ortodóncico; la ausencia o un grosor insuficiente de hueso alveolar corresponde a una complicación para el tratamiento ortodóncico. ${ }^{1}$

El grosor del hueso alveolar define los límites del movimiento ortodóncico, y sobrepasar estos límites puede causar efectos secundarios no deseados para el tejido periodontal como el desarrollo de defectos óseos alveolares, es decir dehiscencias y fenestraciones. 2. Si el área disponible para el movimiento dental se encuentra limitada o reducida, una fuerza ortodóncica excesiva causará la reabsorción de la cortical ósea; los pacientes con defectos óseos alveolares son proclives al desarrollo de recesiones gingivales en estas áreas con la subsecuente exposición radicular, sensibilidad dental y caries radiculares. ${ }^{\mathbf{3} 4}$

Adicionalmente, un contorno alveolar comprometido o inadecuado antes del tratamiento, así como una capacidad limitada de adaptación al movimiento dental, puede restringir o interferir con el movimiento dental potencial o planificado, así como la posición y angulación dental final deseada, llevando a un fracaso en el tratamiento. ${ }^{\mathbf{5}}$

El diagnóstico ortodóncico requiere, por lo tanto, un examen minucioso de los tejidos de soporte periodontal; alteraciones como dehiscencias, fenestraciones y otros defectos óseos deberían ser incluidos tanto dentro del diagnóstico como dentro del plan de tratamiento, puesto que conocer de antemano la presencia de estos defectos podría afectar drásticamente el plan de tratamiento inicial. ${ }^{2}$

La tomografía computarizada (TC) permite al profesional visualizar lo que las radiografías convencionales nunca mostraron: el grosor y la altura del hueso alveolar. ${ }^{2}$. El desarrollo de Tomografías Computarizadas Cone Beam (TCCB) se ha convertido en una gran ayuda en el área de la odontología puesto que permite superar las limitaciones de las radiografías bidimensionales, a un menor costo y a una menor dosis de radiación que las tomografías convencionales.

El tratamiento ortodóncico ha sido catalogado como un potencial generador de defectos óseos; sin embargo, la presencia de estos defectos es mucho más común de lo que pensamos. La visualización de los detalles anatómicos de nuestros pacientes y la comprensión de los efectos secundarios del movimiento dental, va a permitirnos reconocer nuestros límites y a ejercer una ortodoncia más segura. ${ }^{2}$

Considerando las limitaciones de las radiografías bidimensionales en la evaluación de dehiscencias y fenestraciones, la necesidad de su identificación y diagnóstico previo al tratamiento de ortodoncia, y el uso de la TCCB para mejorar el diagnóstico ortodóncico, y además tomando en cuenta la falta de estudios que a la fecha se han realizado sobre el uso de esta tecnología para la detección previa de estos defectos óseos, el propósito de este estudio es investigar la prevalencia y distribución de defectos óseos en un grupo de potenciales pacientes ortodóncicos no tratados, utilizando TCCB, con el objetivo de evaluar su posible incorporación a los estudios diagnósticos básicos previos al tratamiento ortodóncico.

La hipótesis de este trabajo de investigación es que existe una alta incidencia de defectos óseos en pacientes ortodóncicos previo al tratamiento; por lo tanto, es extremadamente importante diagnosticar el estado de la cortical ósea antes de iniciar cualquier tratamiento ortodóncico para detectar la presencia de dehiscencias y fenestraciones, evitar agravarlas durante el tratamiento, y evitar producir efectos no deseados como el desarrollo de recesiones gingivales. 


\section{Métodos}

Este estudio fue llevado a cabo utilizando Tomografías Computarizadas Cone Beam obtenidas a partir de los archivos del Centro Radiológico Xraycenter 3D, una vez obtenida la aprobación del Comité de Bioética de la Universidad San Francisco de Quito.

Рara los fines de este estudio, se analizaron 50 Tomografías Computarizadas Cone Bean de potenciales pacientes ortodóncicos no tratados entre 15 y 35 años de edad, a partir de las cuales se obtuvo una muestra de 33 tomografías siguiendo los siguientes criterios de inclusión: edad entre 15-35 años, dentición permanente, ausencia de agenesias o dientes extraídos, sin tratamiento ortodóncico previo, apiñamiento de leve a moderado, ausencia de enfermedad periodontal, imágenes de alta resolución.

Las TCCB fueron obtenidas a partir de un mismo tomógrafo marca NewTom GiANO con una rotación de $360^{\circ}$ y un voxel de $0,15 \mathrm{~mm}$, utilizando los siguientes ajustes de configuración:

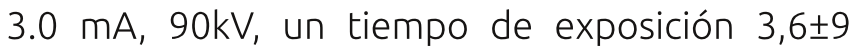
segundos y cortes de $0,15 \mathrm{~mm}$ de grosor. Las imágenes fueron analizadas utilizando el Software de análisis NNT Viewer de NewTom, el cual es el software propio del tomógrafo utilizado.

Todos los dientes, con excepción de segundos y terceros molares fueron incluidos en este estudio, habiéndose analizado un total de 792 dientes.

Se analizó la presencia de dehiscencias y fenestraciones evaluando la superficie vestibular de cada diente en cortes axiales y sagitales. Se consideró defecto óseo o alveolar a cualquier situación en la que no se mostró hueso cortical alrededor de la raíz del diente analizado en 3 vistas consecutivas. Con la herramienta de medición del software de análisis del tomógrafo, se midió la distancia del límite amelocementario a la cresta ósea; si la cresta alveolar se encontraba a una distancia mayor a $2 \mathrm{~mm}$ del límite amelocementario, la situación fue catalogada como una dehiscencia (Figura 1).

Cuando el defecto no incluía la cresta alveolar, el caso fue clasificado como una fenestración (Figura 2).

El modo de reconstrucción 3D fue utilizado para confirmar la presencia de estos defectos óseos.

Posteriormente, con la herramienta de medición del software, se midió el grosor del hueso alveolar vestibular y palatino o lingual en el tercio medio radicular de incisivos centrales, caninos, primeros premolares y primeros molares tanto superiores como inferiores (Figura 3) analizándose un total de 528 dientes y 1056 superficies óseas alveolares. Las mediciones fueron realizadas paralelas al borde inferior del marco del corte tomográfico y fueron ejecutadas por un mismo operador; cada medición fue realizada dos veces y se utilizó el promedio obtenido a partir de las dos mediciones.

Las mediciones obtenidas fueron registradas en una tabla de cálculo digital, excluyéndose cualquier dato personal que permita identificar al paciente (nombre, dirección, etc.). A partir de los datos obtenidos se realizó un estudio estadístico con el fin de cuantificar la incidencia y distribución de dehiscencias y fenestraciones y evaluar el comportamiento del hueso alveolar en cuanto a su grosor.

\section{Resultados}

El 52\% (17 pacientes) de la muestra correspondieron al género femenino, mientras que el $48 \%$ (16 pacientes) al masculino, existiendo una diferencia mínima entre los dos (Gráfico 1)

Un $91 \%$ de los pacientes analizados presentaron al menos un defecto óseo.; así mismo, un 36\% de la muestra presentó algún tipo de defecto óseo (Gráfico 2). Las dehiscencias fueron los defectos óseos más comúnmente encontrados, en comparación con las fenestraciones (Gráfico 3). 
MUESTRA ANALIZADA POR GÉNERO
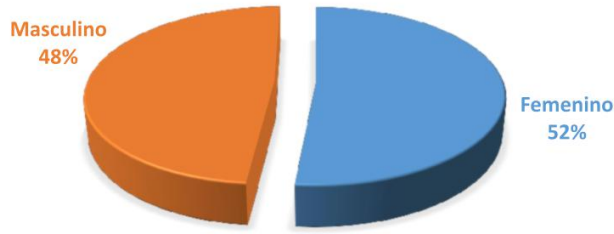

- Femenino Masculino

Gráfico 1. Muestra analizada por género.

Prevalencia de defectos óseos

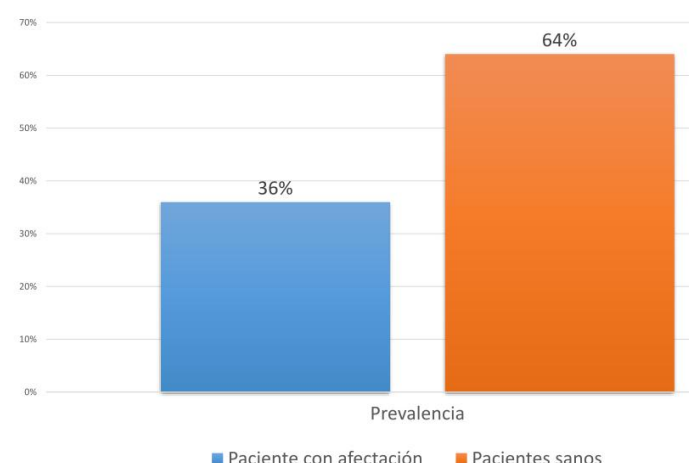

Gráfico 2. Prevalencia de defectos óseos.

Prevalencia de defectos óseos

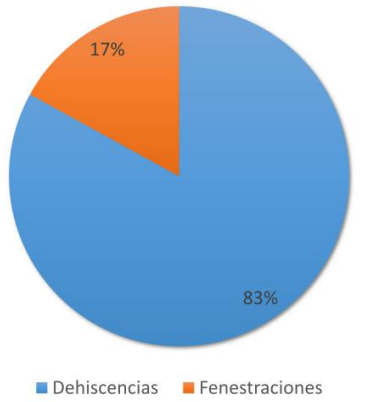

Gráfico 3. Prevalencia de defectos óseos

Las dehiscencias fueron más comunes en la mandíbula (54\%), especialmente en el sector anterior (Tabla 4, Gráfico 5). Al contrario, las fenestraciones fueron más comunes en el maxilar (86\%), especialmente en el sector de premolares (Gráfico 4).

\section{Prevalencia de defectos óseos por arcada}

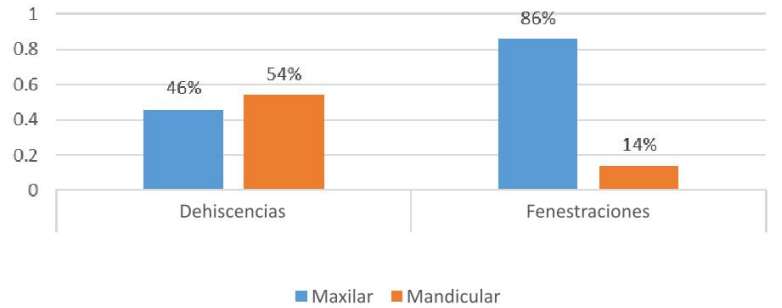

Gráfico 4. Prevalencia de defectos óseos por arcada.

Se observó una mayor prevalencia de defectos óseos en el género femenino, aunque esta relación no fue estadísticamente significativa ( $p>0,05)$ (Gráfico 5).

Prevalencia de defectos óseos por género y arcada dentaria

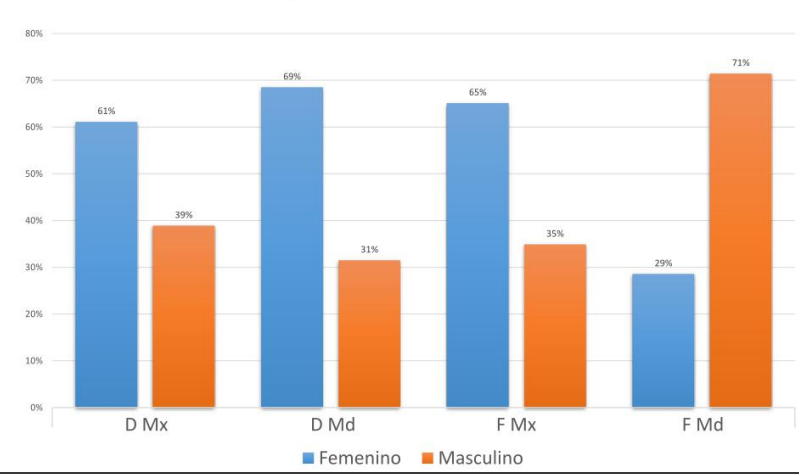

Gráfico 5. Prevalencia de defectos óseos por género y arcada dentaria: DMx (Dehiscencia maxilar), DMd (Dehiscencia mandíbula), FMx(Fenestración maxilar), FMd (Fenestración mandíbula)

Se detectó una mayor prevalencia de defectos óseos a medida que aumentó la edad de la muestra, sin embargo, esta relación fue únicamente significativa con respecto a las dehiscencias $(44,1 \%)(p<0,05)$ (Gráfico 6). 
Prevalencia por de defectos óseos por grupos de edad y arcada dentaria

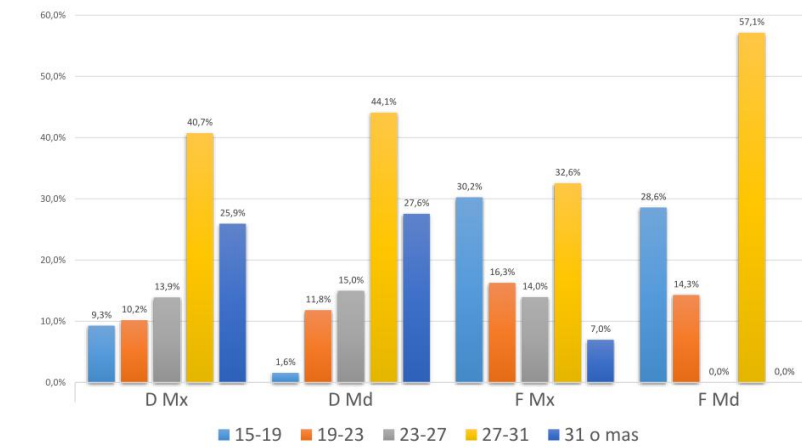

Gráfico 6. Prevalencia de defectos óseos por grupos de edad y arcada dentaria.

Prevalencia de defectos óseos según la pieza dentaria

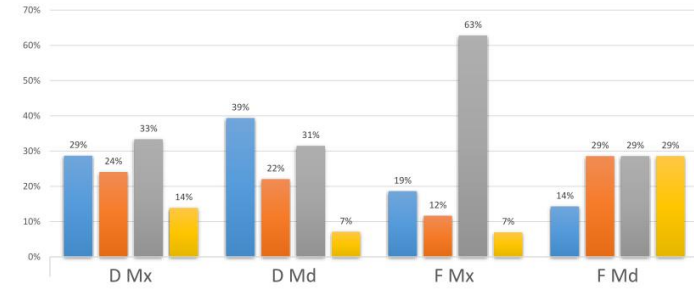

- Incisivo Canino Premolar Molar

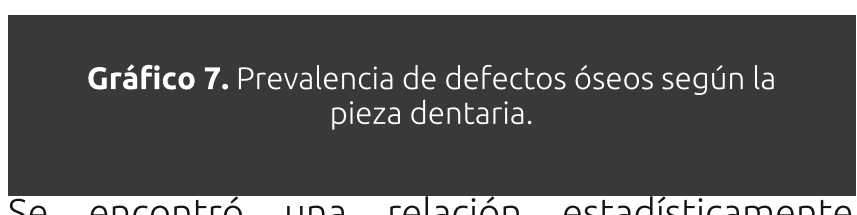

Se encontró una relación estadísticamente significativa entre dehiscencias maxilares y mandibulares $(p<0,05)$

Se confirmó que el mayor grosor de hueso alveolar se encuentra en la superficie lingual de primero molares inferiores, mientras que el más delgado se encuentra en la superficie vestibular de caninos inferiores (Tabla 1).

Este estudio también se enfocó en encontrar si alguna pieza dental en particular, dentro de la muestra estudiada, presentaba mayor afectación de defectos óseos, por lo que se buscó la prevalencia de afectación por tipo de diente, cuyos datos se reportan en el gráfico 7, llegando a la conclusión de que los premolares tanto maxilares como mandibulares fueron las piezas

\begin{tabular}{|l|l|l|l|l|}
\hline DIENTE & $\begin{array}{l}\text { Vestibular } \\
\text { Media en } \mathrm{mm}\end{array}$ & D.S. en $\mathrm{mm}$ & $\begin{array}{l}\text { Palatino/Lingual } \\
\text { Media en mm }\end{array}$ & D.S. en mm \\
\hline $\mathbf{1 6}$ & 1.739 & 0.777 & 1.609 & 0.774 \\
\hline $\mathbf{1 4}$ & 1.091 & 0.761 & 2.133 & 1.518 \\
\hline $\mathbf{1 3}$ & 0.736 & 0.368 & 1.918 & 1.115 \\
\hline $\mathbf{1 1}$ & 0.964 & 0.315 & 2.452 & 1.036 \\
\hline $\mathbf{2 1}$ & 0.848 & 0.336 & 2.227 & 1.276 \\
\hline $\mathbf{2 3}$ & 0.661 & 0.564 & 1.921 & 0.819 \\
\hline $\mathbf{2 4}$ & 1.003 & 0.812 & 1.924 & 1.159 \\
\hline $\mathbf{2 6}$ & 1.639 & 0.812 & 1.561 & 0.841 \\
\hline $\mathbf{3 6}$ & 1.642 & 0.853 & 3.127 & 1.070 \\
\hline $\mathbf{3 4}$ & 0.727 & 0.702 & 3.309 & 1.458 \\
\hline $\mathbf{3 3}$ & 0.370 & 0.283 & 2.042 & 1.052 \\
\hline $\mathbf{3 1}$ & 0.382 & 0.286 & 0.936 & 0.689 \\
\hline $\mathbf{4 1}$ & 0.406 & 0.301 & 0.885 & 0.688 \\
\hline $\mathbf{4 3}$ & 0.355 & 0.310 & 1.833 & 1.130 \\
\hline $\mathbf{4 4}$ & 0.673 & 0.616 & 2.897 & 1.210 \\
\hline $\mathbf{4 6}$ & 1.764 & 0.823 & 3.473 & 1.013 \\
\hline D.S. (Desviación Es tándar) & & & \\
\hline
\end{tabular}

Tabla 1. Medidas de dispersión con respecto al grosor del hueso alveolar.

dentarias que presentaron más defectos óseos. El sector de los premolares maxilares es el que más fenestraciones presentó (63\%), mientras que los incisivos mandibulares son las piezas dentarias que más dehiscencias presentaron (39\%).

En la tabla 1 se muestra el promedio del grosor del hueso alveolar vestibular y palatino/lingual en las distintas piezas dentarias, recordando que, como se mencionó previamente, la medición se realizó en el tercio medio radicular de incisivos centrales, caninos, primeros premolares y primeros molares tanto superiores como inferiores. Cabe resaltar que el valor más alto se presentó en la superficie lingual del primer molar con una media de $3.473 \mathrm{~mm}$ y una DS de $1.013 \mathrm{~mm}$, mientras que el valor más bajo se presentó en la superficie vestibular de caninos inferiores con una media de $0,355 \mathrm{~mm}$ y una DS de 0,310. Tanto en el maxilar como en la mandíbula, el hueso alveolar palatino/lingual presentó mayor grosor que el vestibular. En el maxilar, tanto los caninos como los incisivos presentaron un espesor de hueso vestibular menor a $1 \mathrm{~mm}$. Esta misma situación se presentó en la arcada inferior, sumándose el hueso alveolar vestibular de premolares al grupo de dientes con cantidad de hueso alveolar menor a $1 \mathrm{~mm}$. 
Al cuantificar el Î́ndice de Correlación de Pearson, se pudo verificar que las variables edad y dehiscencias, tanto maxilares como mandibulares, presentaron un p-valor $=0.00<$ 0.05 lo cual implica una relación estadísticamente significativa entre estas dos variables.

Así mismo se observa una relación estadísticamente significante entre dehiscencias maxilares y mandibulares, lo que implica que si encontramos una dehiscencia en una arcada, es probable encontrar dehiscencias en la arcada opuesta.

\section{Discusión}

Después de realizar el análisis estadístico, se ha podido verificar de manera descriptiva e inferencial que, en la muestra estudiada, existió una alta prevalencia de defectos óseos en potenciales pacientes ortodóncicos previo al tratamiento.

Los resultados de este estudio coincidieron con los estudios realizados por Castro y cols, Evangelista y cols, Mostafá y cols., Yagci y cols, Evangelista y cols., y Yang y cols. En el 2012, Yagci y cols. evaluaron la presencia de defectos alveolares en pacientes con maloclusiones I, II y III utilizando TCCB, con el objetivo de determinar si existe una diferencia entre estos 3 grupos. Los autores observaron que las maloclusiones clase II presentaron una mayor incidencia de fenestraciones, y no se encontraron diferencias estadísticamente significativas en la incidencia de dehiscencias. Así mismo, ratificaron que en la mandíbula es más común la presencia de dehiscencias, mientras que en el maxilar es más común encontrar fenestraciones ${ }^{6}$.

Evangelista y cols. evaluaron la presencia de defectos óseos en pacientes con maloclusiones clase I y clase II división 1. Se observó que los defectos óseos son un hallazgo común antes del tratamiento ortodóncico; un $51,09 \%$ de los dientes evaluados (4319) presentaron dehiscencias y un $36,51 \%$ fenestraciones. Además, determinaron que en las maloclusiones clase I existe una mayor incidencia de dehiscencias (35\% más) 7 .

Castro y cols. valoraron la distancia entre el límite amelocementario y la cresta del hueso alveolar antes y después del tratamiento ortodóncico utilizando TCCB. Se encontraron distancias aumentadas en un $57 \%$ de los casos, más comúnmente en las superficies vestibulares y linguales de los incisivos centrales mandibulares. Hallaron dehiscencias óseas en un $11 \%$ de la muestra antes del tratamiento ortodóncico; y un $19 \%$ de las medidas después del tratamiento ortodóncico estuvieron aumentadas; la mayor cantidad de dehiscencias se encontró en la superficie vestibular de caninos superiores y la superficie lingual de incisivos centrales inferiores 8

Yang y cols. examinaron 2574 dientes, de los cuales un 8-6\% presentaron dehiscencias óseas. Estas dehiscencias fueron más frecuentes en la mandíbula que en el maxilar; así mismo, el diente más asociado fue el primer premolar y canino inferiores. Otra conclusión que arrojó este estudio fue que las dehiscencias son más frecuentes en mujeres que en hombres. ${ }^{9}$

Para determinar la incidencia de dehiscencias, en un estudio realizado por Mostafa y cols, se realizó una cirugía de colgajo abierto en el sector anterior mandibular y TCCB de 32 pacientes no tratados, examinando un total de 188 dientes; un $78 \%$ de los participantes presentó al menos un defecto alveolar; las dehiscencias eran los defectos óseos más comunes y fueron más comunes en el tercio medio radicular de la cortical vestibular de caninos inferiores. Esta investigación determinó la necesidad de validar las TCCB para la detección y medición de dehiscencias previo al tratamiento ortodóncico ${ }^{3}$.

La mayor presencia de fenestraciones en la arcada superior, puede deberse a que el tejido 
óseo maxilar es más esponjoso que el mandibular, haciéndolo más propenso a perforaciones. Por el contrario, los resultados de éste y otros estudios concluyen que las dehiscencias son más comunes en la arcada inferior, especialmente en la superficie vestibular del sector anterior, lo cual puede deberse a que este sector es el que presenta la cortical más delgada, haciéndolo más susceptible al desarrollo de dehiscencias. Otra de las posibles razones por lo cual se presentó un porcentaje más elevado de dehiscencias en este sector, puede ser atribuido a la presencia de apiñamiento en la región antero inferior; los sujetos de estudio, al ser potenciales pacientes ortodóncicos, podrían haber presentado en su mayoría apiñamiento anteroinferior, situación que no se evaluó por no ser motivo de nuestro estudio, lo cual podría obligar a ciertos dientes a posicionarse más hacia vestibular, haciéndolos más susceptibles al desarrollo de dehiscencias antes del tratamiento ortodóncico.

La prevalencia de defectos alveolares constituye un dato importante para los ortodoncistas, los cuales pueden informar a los pacientes que las dehiscencias y fenestraciones suelen ser hallazgos comunes en la población y que no son causados únicamente por los movimientos ortodóncicos.

La visualización de los detalles anatómicos de nuestros pacientes y la comprensión de los posibles efectos secundarios de la movilización dentaria nos permitiría reconocer nuestros límites y practicar una ortodoncia segura. Con esto en mente, la indicación de imágenes tomográficas Cone Beam debe ser considerada para pacientes ortodóncicos, especialmente para pacientes mayores a los 27 años.

La TCCB es una herramienta valiosa que permite una planificación del tratamiento ortodóncico en base a la presencia, ubicación y tamaño de dehiscencias y fenestraciones existentes antes del tratamiento, las cuales no pueden ser identificadas utilizando radiografías periapicales, panorámicas, ni cefálicas laterales, las mismas que son utilizadas de manera rutinaria para realizar el diagnóstico ortodóncico. Las mecánicas a utilizar durante el tratamiento deberían tender a reducir el movimiento de los dientes fuera de su cobertura ósea; es muy importante tomar en cuenta este aspecto puesto que en la actualidad es muy popular el uso de técnicas expansivas, las cuales agravarían cualquier problema preexistente. Por otro lado, la comparación de TCCB pre y postratamiento de ortodoncia, ayudarían a determinar si dicho tratamiento produjo o no algún efecto iatrogénico.

Como se mencionó previamente, el detectar defectos óseos previos al tratamiento ortodóncico permite al ortodoncista ejecutar planes de tratamiento alternativos como por ejemplo realizar injertos gingivales previos al tratamiento, decidirse por un tratamiento con extracciones en lugar de un tratamiento expansivo, evitar proinclinar los dientes afectados, utilizar prescripciones con torques selectivos, mantener controles periodontales periódicos durante el tratamiento, utilizar fuerzas ligeras a lo largo de toda la terapia ortodóncica, evitar utilizar aparatología que retenga placa bacteriana, optar por una retención permanente o semipermanente.

Como potenciales desventajas o peros al uso de este tipo de tomografías, se puede enunciar la radiación producida durante la toma de la TCCB y el valor elevado de estos exámenes; sin embargo, si tomamos en cuenta que la TCCB puede reemplazar a la radiografía panorámica y a la radiografía cefálica lateral, usadas en forma rutinaria como parte del diagnóstico ortodóncico y a la radiografía oclusal y radiografías periapicales, usadas en los casos en los que se requiere profundizar el estudio de una zona específica, para realizar un diagnóstico apropiado, el costo biológico por la radiación emitida y el costo económico de este conjunto de radiografías puede ser muy próximo al costo biológico y económico de una TCCB, que sumado 
a las ventajas de poder visualizar las estructuras desde todos los ángulos, poder realizar cortes y mediciones, poder diagnosticar la presencia de defectos óseos, como se ha comprobado en este estudio, es decir, poniendo en la balanza costo tanto biológico como económico, versus beneficio, justifica su indicación en lugar del conjunto de radiografías mencionadas previamente.

\section{Conclusiones}

Los cortes axiales y sagitales de las TCCB permiten observar un panorama general del grosor del hueso alveolar vestibular y lingual y permitieron identificar que los defectos óseos corresponden a un hallazgo común en potenciales pacientes ortodóncicos no tratados.

Las TCCB poseen el potencial de reemplazar las radiografías convencionales y permitir realizar un diagnóstico más completo que permita a su vez tomar decisiones terapéuticas acertadas en base a la arquitectura ósea de cada paciente, por lo que se recomienda su uso como auxiliar diagnóstico, especialmente en pacientes de alto riesgo, es decir pacientes adultos, con un biotipo periodontal delgado, que requieran un tratamiento ortodóncico expansivo.

\section{Referencias Bibliográficas}

1. Gamba, D., \& etal. Periodontal effects of rapid maxillary expansion with toothtissue-borne and tooth-borne expander: a computed tomography evaluation. AJODO. 2010; 15(5):192-205.

2. Mostafa, Y., \& etal. Do alveolar bone defects merti orthodontists respect?. World Journal of Orthodonics. 2009; 10(1):16-20.

3. Pudyani, P., \& etal. Morphological changes of alveolar bone due to orthodontic movement of maxillary nd mandibulary incisors. Dent. J. 2008; 41(1): 21-24.
4. Kapila, S., \& Nervina, J. СВCT in orthodontics: assessment of treatment outcomes and indications for its use. Dentomaxillofacial radiology. 2015; 44: 119.

5. Ising, $N$. The evaluation of dehiscences using cone beam computed tomography (Tesis de maestría). Saint Louis: Graduate School of Saint Luois University. 2010.

6. Yagci, A., \& etal. Dehiscence and fenestrarion in skeletal Class I, II, and III malocclusions assessed with cone.beam computed tomography. Angle Orthodontis. 2012; 82: 67-74.

7. Evangelista, K., \& etal. Dehiscence and fenestration in patients with Class I and Class II division 1 malocclusiones assessed with cone-beam computed tomography. AJODO. 2010; 138(2):133.e1-133.e7.

8. Castro, L., \& etal. Cone beam computed tomography evaluation of distance from cementoenamel junction to alveolar crest before and after nonextraction orthodontic treatment. Angle Orthod. N.f; $0(0): 1-7$

9. Yang, Y., \& etal. Evaluation and New Classification of Alveolar Bone Dehiscences Using Cone-Beam Computed Tomography in vivo. Int. J. Morphol. 2015; 33(1): 361-368. 